\title{
Gender inequality and sex differences in physical fighting, physical activity, and injury among adolescents across 36 countries
}

Margaretha de Looze, Frank J. Elgar, Candace Currie, Petra Kolip, and Gonneke W. J. M. Stevens

\begin{tabular}{|l|l|}
\hline Date of deposit & 05112018 \\
\hline Document version & Author's accepted manuscript \\
\hline Access rights & $\begin{array}{l}\text { Copyright C } 2018 \text { Society for Adolescent Health and Medicine. All } \\
\text { rights reserved. This work is made available online in accordance } \\
\text { with the publisher's policies. This is the author created, accepted } \\
\text { version manuscript following peer review and may differ slightly } \\
\text { from the final published version. }\end{array}$ \\
\hline $\begin{array}{l}\text { Citation for } \\
\text { published version }\end{array}$ & $\begin{array}{l}\text { de Looze, M., Elgar, F. J., Currie, C., Kolip, P., \& Stevens, G. } \\
\text { (2019). Gender inequality and sex differences in physical fighting, } \\
\text { physical activity, and injury among adolescents across 36 } \\
\text { countries. Journal of Adolescent Health, 64(5), 657-663. }\end{array}$ \\
\hline $\begin{array}{l}\text { Link to published } \\
\text { version }\end{array}$ & \begin{tabular}{l} 
https://doi.org/10.1016/j.jadohealth.2018.11.007 \\
\hline
\end{tabular}
\end{tabular}

Full metadata for this item is available in St Andrews Research

Repository at: https://research-repository.st-andrews.ac.uk/

\section{St Andrews Research Repository}


Abstract

Purpose: Sex differences in adolescent health are widely documented, but social explanations for these sex differences are scarce. This study examines whether societal gender inequality (i.e., men's and women's unequal share in political participation, decision-making power, economic participation and command over resources) relates to sex differences in adolescent physical fighting, physical activity, and injuries.

Methods: National-level data on gender inequality (i.e. the United Nations Development Program's Gender Inequality Index) were linked to health data from 71,255 15-year olds from 36 countries in the 2009/10 Health Behavior in Schoolaged Children (HBSC) study. Using multilevel logistic regression analyses, we tested the association between gender inequality and sex differences in health while controlling for country wealth (GDP per capita).

Results: In all countries, boys reported more physical fighting, physical activity, and injuries than girls, but the magnitude of these sex differences varied greatly between countries. Societal gender inequality positively related to sex differences in all three outcomes. In more gender unequal countries, boys reported higher levels of fighting and physical activity, compared to boys in more gender equal countries. In girls, scores were consistently low for these outcomes, however injury was more common in countries with less gender inequality. 
Conclusions: Societal gender inequality appears to relate to sex differences in some adolescent health behaviors and may contribute to the establishment of sex differences in morbidity and mortality. To reduce inequalities in the health of future generations, public health policy should target social and cultural factors that shape perceived gender norms in young people.

Implications and contribution Societal gender inequality relates to sex differences in physical fighting, activity and injuries among adolescents. That gender inequality is reflected in adolescent health behaviors suggests that gender inequalities in health and health behaviors are, at least partially, socially constructed.

Key words: gender inequality; adolescence; physical fighting; physical activity; injury; Europe; North America. 
Sex inequalities exist in many aspects of health in adolescence in high-income countries [1]. One of the most established findings in adolescent health research is that girls report lower well-being (e.g., lower life satisfaction, more health complaints) than boys [1-3], while boys engage more in active and externalizing behaviors than girls (e.g., physical fighting, physical activity, injuries [4-6]). Although there is some consistency in the gendered patterning of these outcomes, the size of the sex differences varies across countries, with larger differences found in Eastern Europe, and smaller differences in Scandinavian countries [1]. The cross-national variability in the sex differences in adolescent well-being and behaviors suggests that contextual factors reinforce the gendered nature of health during adolescence. Based on the assumption that societal gender inequality reflects the extent to which boys and girls are expected to behave in ways that are consistent with traditional gender norms (i.e., boys should be active and aggressive, while girls should be passive and submissive; [7]), this study examines whether sex differences in adolescent physical fighting, physical activity, and injuries relate to societal gender inequality.

Societal gender inequality, defined as men's and women's unequal share of paid work, educational level, health and political decision-making power in society as per the Gender Inequality Index (GII), varies considerably across Europe [8]. Research indicates a link between societal gender inequality and prevailing traditional gender norms within countries $[9,10]$. This link may exist either because culturally dominant gender norms increase pressure for a decrease in societal gender inequality or because societal gender equality triggers an emancipatory shift in norms [11]. From a social psychological framework, gender norms function to guide behavior by providing information about what is 
considered "normal" or "appropriate" behavior for men and women in a given society [5,12-15]. To illustrate, if girls observe female role models who are educated, independent, and powerful beings in society, they may be less likely to adhere to traditional gender norms of passivity and submissiveness and more confident to take risks and to engage in so-called 'masculine' behaviors. As such, smaller sex differences in these behaviors can be expected in countries with lower societal gender inequality.

Physical fighting and engaging in physical activity, with injuries as a potential consequence of both [16-17], are prominent examples of behaviors that are traditionally regarded as masculine. Both at an individual [18] and national [19] level, traditional gender norms relate to more engagement in sports by men and less engagement by women. It has been suggested that men use sports to acquire a more muscular and masculine appearance, while women shy away from sports because they want to appear feminine [18]. Violent behaviors also relate to holding traditional gender norms, especially among men [20], possibly because violence can be seen as an expression of masculine dominance [21]. It has been suggested that males may feel compelled to fight in order to feel masculine or prove themselves masculine to others [22]. As adolescence is a life stage in which age-appropriate gender identities are actively (re)formed and the desire to adhere to social norms and 'do gender well' is high [23] -i.e., deviating from the norms associated with one's biological sex can be met with censure from peers and social exclusion, and sometimes even emotional or physical violence [5] adolescents, more than any other age group, may use behaviors such as physical fighting and physical activity to reflect and adopt norms of society with respect to gender $[7,15,24,25]$. 
To our knowledge, only one study addressed the link between societal gender inequality and sex differences in adolescent health. Torsheim and colleagues [2] examined the association between societal gender inequality and sex differences in mental health (i.e., subjective health complaints). While subjective health complaints were more prevalent among girls than boys in most European and North American countries, sex differences were larger in countries with high gender inequality. To date, however, it is unknown if gender inequality relates to health behaviors that would be specifically encouraged for one gender and discouraged for the other based on traditional gender norms. If societal gender inequality affects the extent to which adolescent boys and girls engage in physical fighting, physical activity, and experience injuries, then it contributes to inequalities in adolescent (and eventually, adult) morbidity and mortality. Addressing this issue is a top priority for the health and well-being of current and future generations in the light of the 2030 Agenda for Sustainable Development [26].

\section{The present study}

We examined the association between national-level gender inequality and individual-level sex differences in adolescent physical fighting, physical activity, and injuries in 36 countries in Europe and North America. Specifically, we addressed the following research questions: 1) To what extent do sex differences in adolescent physical fighting, physical activity and injuries vary across European and North American countries? 2) Do these sex differences relate to national-level gender inequality? 
Descriptive, cross-national comparative research [1] suggests that adolescent physical fighting, physical activity and injuries are more common among boys, compared to girls, and that considerable country-level differences exist. Based on the assumption that societal gender inequality reflects the extent to which boys and girls are expected to behave in ways that are consistent with traditional gender norms, we hypothesized that sex differences in adolescent physical fighting, physical activity and injury positively relate to national-level gender inequality.

\section{Method}

\section{Study Population and Procedures}

Data were obtained from the WHO collaborative Health Behavior in School-aged Children (HBSC) Study 2009/2010. Between Fall 2009 and Spring 2010, anonymous surveys were conducted in the classrooms of 11-, 13- and 15year olds according to a common research protocol [27]. Each participating country obtained approval to conduct the survey from the ethics review board or equivalent regulatory body associated with their respective institutions/countries. Participation was voluntary and informed consent was sought from school administrators, parents and children according to local human subject requirements. School response rates varied by country but were $>70 \%$ in most countries. At the student-participant level, response rates ranged from 44 to $92 \%$, but they were $>70 \%$ in almost all countries. For more information on study procedures, see [28].

Country-level data on the UNDP Gender Inequality Index (GII; 29) were linked with the HBSC data. For this purpose, the HBSC samples from the French 
and Flemish speaking parts of Belgium were merged and weighted according to their populations of 15 -year-olds [30]. We did the same with the samples from England, Scotland and Wales (UK sample). Greenland was excluded from the analyses because the GII for Denmark only refers to its mainland. As sex differences in health are generally largest in 15-year olds [31], only 15-year-olds were included in the current analysis. This resulted in a sample of $N=71,255$ adolescents (49.5\% females) in 36 countries.

\section{Measures}

\section{Country-level variables}

Gender Inequality Index. We used the Gender Inequality Index (GII) provided by the United Nations Development Program (UNDP, 2011) as a measure for national-level gender inequality. The GII was first published in 2010 and has replaced the Gender Development Index (GDI) and the Gender Empowerment Measure (GEM) that both had limitations [32-33]. The GII is based on statistical data on gender-based differences in the three dimensions of reproductive health (i.e., maternal mortality ratio and adolescent birth rates), empowerment (i.e., proportion of parliamentary seats occupied by females and proportion of adult females and males aged 25 years and older with at least some secondary education) and economic status (i.e., labour force participation rate of females and males aged 15 years and older). GII ranges theoretically from 0 (indicating that women and men are equal) to 1 (indicating greatest levels of inequality, i.e., that women fare as poorly as possible in all measured dimensions).

GDP per capita. As most countries with high levels of gender equality are relatively wealthy [9], the gross domestic product (GDP) per capita was included 
as a control variable in the analyses. This way, we could rule out that identified associations in this study reflected links with high national wealth, rather than with gender equality.

\section{$\underline{\text { Individual-level variables }}$}

Physical fighting. Physical fighting was measured with the following question:

"During the past 12 months, how many times were you in a physical fight?" Answer categories were never, once, twice, three times, and four or more times. To identify young people for whom violence is likely habitual as opposed to an occasional or nonexistent behavior, this variable was dichotomized to measure frequent fighting behavior ( $0=$ less than four times; $1=$ four times or more).

Physical activity. Physical activity was measured with the following question: "Over the past 7 days, on how many days were you physically active for a total of at least 60 minutes per day?" Answer categories ranged from 0 days to 7 days. The item is preceded by a common definition: "Physical activity is any activity that increases your heart rate and makes you get out of breath some of the time. Physical activity can be done in sports, school activities, playing with friends, or walking to school. Some examples of physical activity are running, brisk walking, inline skating, biking, dancing, skateboarding, swimming, soccer, basketball, and football." Based on the current worldwide recommendation of 60 minutes of physical activity per day [1,5], this variable was dichotomized to measure frequent physical activity, with $0=$ less than seven days and $1=$ seven days.

Injuries. Adolescents were asked: "During the past 12 months, how many times were you injured and had to be treated by a doctor or nurse?” Answer 
categories were never, once, twice, three times, and four or more times. As per existing precedents $[1,31]$, response categories were dichotomized with $0=$ never and $1=$ at least once.

\section{Statistical Analyses}

First, we assessed the prevalence of the outcomes, separately for boys and girls at the country level. In countries where weights were provided by the national team these weights were taken into account, as well as weights for the national samples for Belgium and Great Britain, as explained above. Next, we calculated absolute differences in the prevalence of frequent physical fighting, daily physical activity, and injuries between boys and girls in each country, and tested correlations between these differences and the GII.

Subsequently, multilevel logistic regression models were applied to test the association of gender, gender inequality, and their interaction on each health outcome whilst controlling differences in GDP per capita and other unmeasured differences at the national level. These analyses were performed using Stata/SE 13 (College Station, Texas, USA). Predicted scores on each outcome were estimated using Stata's margins command. Finally, these analyses were repeated with a nominal GII variable, for which countries were grouped based on their GII (tertiles of low, medium, and high scores). Goodness-of-fit of these models were evaluated using the Akaike Information Criterion (AIC) - a measure of model deviance (d) adjusted for the number of parameters ( $q$ ) in the model $(A I C=d+2 q)$, and the more conservative Bayesian Information Criterion (BIC), which also corrects for differences in the number of observations (n) in the model $(B I C=d+\log (n) \times q)[34]$. Smaller AIC and BIC values indicate better model fit. 
Insert Table 1 over here

\section{Results}

Table 1 gives an overview of the sample sizes, gender distribution, and GII for all countries included in our analysis. The GII ranged from a low of .049 (Sweden) to a high of .443 (Turkey). Table 1 also presents the estimated prevalence of frequent physical fighting, daily physical activity, and injuries, separately for boys and girls. Boys in all countries were more likely than girls to engage in all outcomes. Prevalence rates for all outcomes varied considerably across countries. For frequent physical fighting, percentages ranged from 3.8\% (Germany) to $43.5 \%$ (Armenia) among boys and from $0.8 \%$ (Israel) to $5.9 \%$ (Greece) for girls. The magnitude of absolute sex differences ranged from $2.4 \%$ in Germany to $38.8 \%$ in Armenia.

The percentage of boys engaging in physical activity seven days a week ranged from $11.5 \%$ (Italy) to $33.4 \%$ (USA) for boys and from $4.9 \%$ (Italy) to $16.9 \%$ (USA) for girls. The magnitude of absolute sex differences ranged from $3.8 \%$ in Norway to $16.9 \%$ in Spain. For injuries, percentages varied from $16.8 \%$ (Macedonia) to $66.2 \%$ (Spain) for boys and from $8 \%$ (Macedonia) to $56.2 \%$ (Spain) for girls. The magnitude of absolute sex differences ranged from $1.0 \%$ in Norway to $17.3 \%$ in Italy.

The Pearson correlation of GII with the national magnitude of sex differences in adolescent outcomes was strongest for injuries $(r=.284, p=.001)$, 
followed by frequent fighting $(r=.279, p=.001)$ and daily physical activity ( $r$ $=.195, p=.007$ ). When Armenia (as a country with an extremely high prevalence of fighting) was excluded from the analysis, the correlation between the GII and frequent physical fighting remained high $(r=.251, p=.002)$.

Table 2 shows the results of the multilevel logistic regression analyses. With country differences in GDP per capital held constant, girls were significantly less likely to engage in physical fighting, physical activity, and to experience injuries than boys. Furthermore, overall, physical fighting and physical activity were higher in countries with more gender inequality. For all three outcomes, there was a significant interaction between adolescent gender and gender inequality $(p \mathrm{~s}<.001)$, indicating that sex differences in physical fighting, physical activity, and injuries are larger in countries with more gender inequality, compared to countries with less gender inequality.

The interaction effects are further illustrated by a presentation of the marginal effects of gender on physical fighting, physical activity, and injuries for countries at low, medium, and high gender inequality (Table 3 and Figure 1). Table 3 shows that although the odds ratios of gender on the three outcomes are significant for the three groups of countries, the associations are weaker in the countries with lower gender inequality. Figure 1 indicates that physical fighting and physical activity among boys increase as gender inequality increases, while physical fighting and physical activity rates among girls remain low, irrespective of the level of gender inequality. Additionally, girls are less likely to be injured as gender inequality increases, while for boys the prevalence of injuries hardly differs between countries at low, medium and high gender inequality. 
Insert Table 3 and Figure 1 about here

\section{Discussion}

Sex differences in adolescent health behaviors are widely documented, but explanations for these sex differences are scarce. This study aimed to explain cross-national variation in the magnitude of sex differences in adolescent physical fighting, physical activity, and injuries across Europe and North America by focusing on national-level gender inequality. First, our findings indicated that boys reported more fighting, physical activity, and injuries than girls in all countries, although the magnitude of the sex differences varied strongly between countries. Secondly, sex differences were larger in countries that have more national gender inequality. By grouping the countries into low, medium and high gender inequality, it was found that boys engage most in typically male behaviors such as fighting and physical activity in countries high in gender inequality, while scores of girls were consistently low. In girls, the prevalence of injuries was the highest in countries with low gender inequality.

These results suggest that societal gender inequality is reflected in adolescent health behaviors and outcomes at the individual level. From a socialconstructionist perspective, adolescents act in accordance with normative expectations that reinforce gender roles $[15,24,26]$. In countries where traditional gender norms prevail, adolescent boys may exhibit more masculine behaviors such as physical fighting and physical activity, while girls in these countries behave less in these behaviors. At the same time, women in these countries may 
encounter more difficulties regarding their position in the labour market, their reproductive health and possibilities for empowerment (as per the definition of the GII).

While we are cautious about causal conclusions given the correlational nature of the data, another explanation for the identified links is that gender inequality impacts young people's behavior through societal restrictions on boys' and girls' behavior. Girls in countries with high gender inequality tend to have fewer opportunities and choices to shape their personal lives than boys [35]. Constraints on girls' chances to engage in specific behaviors may reinforce sex differences in adolescent health behaviors and subsequently more structural inequalities in workforce participation, empowerment, and reproductive rights. Further examination of the barriers faced by women and girls remains an important area for further research.

The relationship between gender inequality and the health of adolescent boys and girls is complex: boys in more gender-unequal countries fight more, which brings along negative health consequences, but they also engage more in physical activity, and girls are less likely to be injured, which are positive health outcomes. Moreover, our results suggest that injuries are not merely the result of physical fighting and physical activity; while gender inequality affects especially boys when it comes to physical fighting and physical activity, girls are the ones affected when it comes to injuries. Potentially, in more gender-equal countries, girls feel more free to engage in risky behaviors, such as climbing trees and using substances, which also increases their risk of injury, without necessarily engaging in aggressive behaviors like fighting. Girls' engagement in physical fighting was extremely low across countries, indicating that there may either be a biological 
difference between the genders regarding aggression [36], or aggressive behaviors are less accepted for girls even in the most gender-equal societies.

The findings of this study are relevant for the public health domain because they suggest that social contextual factors influence adolescent health behaviors in ways that increase social inequalities in health. With research estimating that $50 \%$ of morbidity and mortality is due to modifiable health behaviors [37], gender inequality at a societal level may eventually contribute to the establishment of sex differences in morbidity and mortality among adolescents as well as adults. Societal gender inequality may function as a barrier to change and may prevent equalisation in adolescent health (behaviors) between genders. Decreasing health risks for boys and girls thus requires far more than simply implementing mass media campaigns aimed at convincing girls to become more physically active and convincing boys to stop fighting. Public health policy can adopt a society-level perspective in addressing gender norms and health disparities by eliminating gender biases in public health campaigns. For example, a recent Canadian tv campaign about preventing head injury in young hockey players only showed girls. It thereby implicitly stated that playing hockey and having injuries do not reflect boys' experiences only.

Limitations of the study should noted. First, our data remain inherently correlational in nature and do not support causal conclusions. However, given that we measure gender inequality as reported by the UNDP (and not adolescents themselves), we avoid some potential problems of endogeneity (i.e., health behaviors influencing adolescents' perceptions of gender inequality). A second limitation is a potential selectivity bias in our sample due to our use of complete case analysis. Given that non-respondents are likely from higher risk groups, we 
possibly have slightly inflated estimates of adolescent physical activity, and slightly lower levels of fighting and injury. Reducing variability in the sample may result in an underestimation of the association between gender inequality and adolescent behavior. Third, our outcome variables were single-item measures, which may misclassify some individuals, leading to bias or error. For example, our outcome variable of injury was broadly defined. Rather than focusing on severe injuries, we included any type of injury adolescents had experienced for which they received medical attention (irrespective of the cause). This broad definition of injury likely biased our results toward no effect. Similarly, adolescents who exercised at a daily basis but less than one hour per day were not classified as adolescents who engage in daily physical activity. This may have led to an underestimation of physical activity in this study. Finally, due to the limited availability of measures in the HBSC dataset, we were not able to test potential mechanisms through which societal gender inequality might affect behavior in adolescent boys and girls (e.g., individual adherence to traditional gender norms; parenting behaviors). If future datasets become available that include such measures, then replication of our analyses including these factors is warranted. Acknowledging these limitations, this study fills an important gap in the literature, as it offers an explanation for the widely documented, but largely unexplained variations in sex differences in adolescent health behaviors across countries. The use of large, nationally representative samples and the inclusion of 36 countries increase the generalizability of our findings. By applying multilevel analyses, we took into account the hierarchical clustering of individuals within countries. Finally, the simultaneous inclusion of economic (GDP per capita) and 
social (GII) national factors is innovative; previous studies have rarely analysed social and economic indicators of health and well-being in a single design.

In conclusion, this study shows that adolescent sex differences in physical fighting, physical activity, and injuries positively relate to national-level gender inequality. Societal gender inequality may encourage boys and girls to behave in ways that are consistent with traditional gender norms. The results of this study speak to the interdependency of UN SDGs 3 (i.e., ensure healthy lives and promote well-being for all at all ages), 5 (i.e., achieve gender equality and empower all women and girls), and 10 (i.e., reduce inequality within and among countries). To reduce sex differences in adolescent health and improve the health of future generations, public health policy should adopt a society-level perspective in addressing gender norms and disparities. 


\section{References}

[1] Inchley J, Currie D, Young T, Samdal O, Torsheim T, Augustson L, et al. (eds.).

Growing up unequal: gender and socioeconomic differences in young people's health and well-being. Health Behavior in School-aged Children (HBSC) study: international report from the 2013/2014 survey. Copenhagen: WHO Regional Office for Europe; 2016.

[2] Torsheim T, Ravens-Sieberer U, Hetland J, Välimaa R, Danielson M, Overpeck M. Cross-national variation of gender differences in adolescent subjective health in Europe and North America. Soc Sci Med 2006; 62: 815-827.

[3] Thapar A, Collishaw S, Pine DS, Thapar A. Depression in adolescence. Lancet 2012; 379(9820): 1056-67.

[4] de Looze M, Pickett W, Raaijmakers Q, Kuntsche E, Hublet A, Nic Gabhainn S, et al. Early risk behaviors and adolescent injury in 25 European and North American countries: A cross-national consistent relationship. J Early Adol 2012; 32: 104-25.

[5] Hallal PC, Andersen LB, Bull F, Guthold R, Haskell W, Ekelund U. Global physical activity levels: surveillance progress, pitfalls, and prospects. Lancet 2012; 380(9838): 247-257.

[6] Pickett W, Molcho M, Elgar FJ, Brooks F, de Looze M, Rathmann K, et al. Trends and socioeconomic correlates of adolescent physical fighting in 30 countries. Pediatrics 2013; 131: 1-9.

[7] Courtenay WH. Constructions of masculinity and their influence on men's well-being: a theory of gender and health. Soc Sci Med 2000; 50:1385-1401.

[8] UNDP. Human Development Report 2011. United Nations; 2017.

[9] Inglehart R, Norris P. Rising Tide: Gender Equality and Cultural Change around the World. New York: Cambridge University Press; 2003.

[10] Schwartz SH, Rubel-Lifschitz T. Cross-national variation in the size of sex differences in values: Effects of gender equality. J Personality Soc Psych 2009; 97(1): 171-185.

[11] Alexander, A. C., \& Welzel, C. (2015). Eroding patriarchy: The co-evolution of women's rights and emancipative values. International Review of Sociology, 25, 144-165

[12] Hawkes S, Buse K. Gender and global health: evidence, policy, and inconvenient truths. Lancet 2013; 381: 1783-87. 
[13] Mahalik JR, Burns SM, Syzdek M. Masculinity and perceived normative health behaviors as predictors of men's health behaviors. Soc Sci Med 2007; 64: 22012209.

[14] Viner, RM, Ozer EM, Denny S, Marmot M, Resnick M, Fatusi A et al. Adolescence and the social determinants of health, Lancet 2012; 379 (9826): 1641-1652.

[15] West C \& Zimmerman D. Doing gender. Gender \& Society 1987; 1: 125-151.

[16] Janssen I, Dorstaler S, Boyce W, Pickett W. Influence of Multiple Risk Behaviors on Physical Activity-Related Injuries in Adolescents. Pediatrics 2007; 119: e672-e680.

[17] Pickett W, Craig W, Harel Y, Cunningham J, Simpson K, Molcho M, et al. Crossnational study of fighting and weapon carrying as determinants of adolescent injury. Pediatrics 2005;116(6):e855-e863.

[18] Spencer RA, Rehman L, Kirk SF: Understanding gender norms, nutrition, and physical activity in adolescent girls: a scoping review. Int J Behav Nutr Phys Act 2015, 12:6.

[18] Van Tuyckom C, Van de Velde S, Bracke P. Does country-context matter? A crossnational analysis of gender and leisure time physical inactivity in Europe. Eur J Pub Health 2013; 23(3): 452-457.

[20] Fleming PJ, Agnew-Burne C. Current trends in the study of gender norms and health behaviors. Curr Opin Psychol 2015; 5: 72-77.

[21] Poteat, V. P., Kimmel, M. S., \& Wilchins, R. (2011). The moderating effects of support for violence beliefs on masculine norms, aggression, and homophobic behavior during adolescence. Journal of Research on Adolescence, 21, 434-447.

[22] Kupers, T. A. (2005). Toxic masculinity as a barrier to mental health treatment in prison. Journal of Clinical Psychology, 61, 713-724.

[23] MacLean A, Hunt K, Sweeting H. Symptoms of Mental Health Problems: Children's and Adolescents' Understandings and Implications for Gender Differences in Help Seeking. Children \& Society 2013; 27(3): 161-173.

[24] Kimmel MS. The Gendered Society, $3^{\text {rd }}$ ed. New York: Oxford University Press; 2008.

[25] UNICEF. Early gender socialization, http://www.unicef.org/earlychildhood/index_40749.html [accessed 01.03.18].

[26] WHO (2015). Transforming our world: the 2030 agenda for sustainable development, 
https://sustainabledevelopment.un.org/post2015/transformingourworld [accessed 01.03.18].

[27] Currie C et al. (eds.) Health Behavior in School-aged Children (HBSC) study protocol: Background, Methodology and Mandatory Items for the 2009/2010 Survey. Edinburgh: CAHRU; 2011.

[28] Roberts C, Freeman J, Samdal O, Schnohr CW, de Looze ME, Nic Gabhainn S, Iannotti R, Rasmussen M. The Health Behavior in School-aged Children (HBSC) study: Methodological developments and current tensions. International Journal of Public Health 2009; 54: S140-150.

[29] UNDP. Human Development Report 2011. United Nations; 2011.

[30] Eurostat. Population on 1 January by age and sex - NUTS 2 regions. 2012, http://appsso.eurostat.ec.europa.eu/nui/show.do?dataset=demo_r_d2jan

[31] Currie C, Zanotti C, Morgan A, Currie D, de Looze M, Roberts C et al. Social determinants of health and well-being among young people. Health Behavior in School-aged Children (HBSC) Study: International Report from the 2009/10 Survey. Health Policies for Children and Adolescents, No. 6. Copenhagen: WHO; 2012.

[32] Benería L, Permanyer I. The Measurement of Socio-Economic Gender Inequality Revisited. Development and Change 2010; 41(3): 375-99.

[33] Dijkstra AG. Revisiting UNDP's GDI and GEM: Towards an alternative. Soc Ind Res 2002; 57: 301-338.

[34] Burnham, K. P., \& Anderson, D. R. (2002). Model selection and multimodel inference: a practical information-theoretic approach (2nd ed.), Springer: New York

[35] Bird CEA, Rieker PP. Gender and Health: The Effects of Constrained Choice and Social Policies. New York: Cambridge University Press; 2008.

[36] Montoya ER, Terburg D, Bos PA, van Honk J. Testosterone, cortisol, and serotonin as key regulators of social aggression: A review and theoretical perspective. Motivation and Emotion 2012; 36(1): 65-73.

[37] Mokdad AH, Marks JS, Stroup DF, Gerberding JL. Actual causes of death in the United States, 2000. JAMA 2004; 291(10):1238-45. 